\section{A seventeen-year-old female with hepatosplenic T-cell lymphoma associated with parvoviral infection}

\author{
Saadiya A. Haque, ' Ying Xiang,' \\ Metin Ozdemirli,' Aziza Shad,' \\ Bhaskar Kallakury' \\ 'Department of Pathology; \\ 2Department of Pediatric Oncology, \\ Georgetown University Hospital, \\ Washington DC, USA
}

\begin{abstract}
Hepatosplenic T-cell lymphoma (HSTL) is rare, being derived from cytotoxic T-cells, and manifests as an extranodal systemic lymphoma. We present an unusual case of a seventeen-year-old female, with no significant prior medical history, presenting with a hepatosplenic T-cell lymphoma. The diagnosis was confirmed by histological examination, immunohistochemisty, and flow cytometry. A staging work-up demonstrated bone marrow involvement by HSTL with concomitant intranuclear parvoviral inclusions.
\end{abstract}

\section{Introduction}

Hepatosplenic T-cell lymphoma (HSTL) is a rare entity, comprising less than $1 \%$ of all non-Hodgkin lymphomas. ${ }^{1}$ It manifests as an extranodal systemic lymphoma, derived from cytotoxic T-cells. The neoplasm is seen mostly in adolescents and young adults with a strong predilection for males $(86 \%) .{ }^{1}$ Twenty percent of HSTL is associated with solid organ transplantation, and usually presents as a post-lymphoproliferative disorder. ${ }^{2}$ It also occurs in children who are on long-term immunosuppressive therapy for Crohn's disease. ${ }^{3}$ HSTL has been reported in the context of EBV or HBV infections. ${ }^{4}$ We present an unusual case of an adolescent female with HSTL and previously unreported concomitant parvoviral infection.

\section{Case Report}

A seventeen-year-old female, with a twoyear history of axillary hidradenitis treated with surgery and antibiotics, presented with a three-day complaint of right thigh pain with fever. Laboratory data revealed severe thrombocytopenia (42 k/uL), mild microcytic

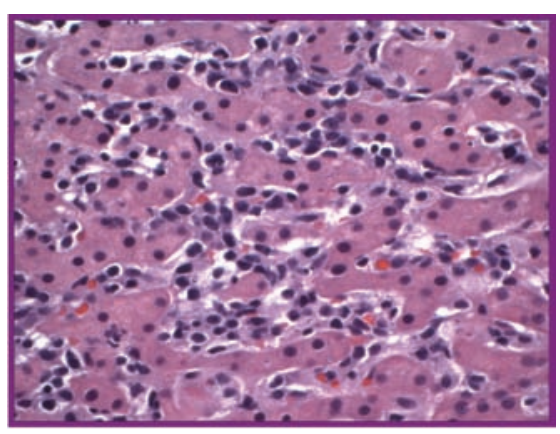

Figure 1. Liver with diffuse sinusoidal infiltration by neoplastic T-cells (hematoxylin and eosin stain; 200X magnification).

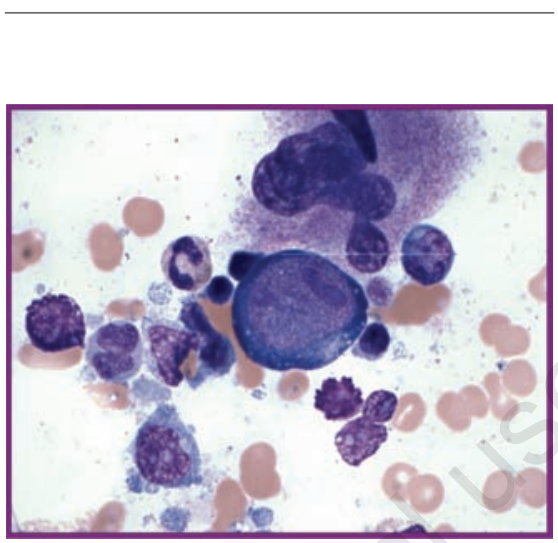

Figure 2. Parvoviral nuclear inclusion in an erythroid precursor seen in a bone marrow aspirate (Wright-Giemsa stain; $400 \mathrm{X}$ magnification).

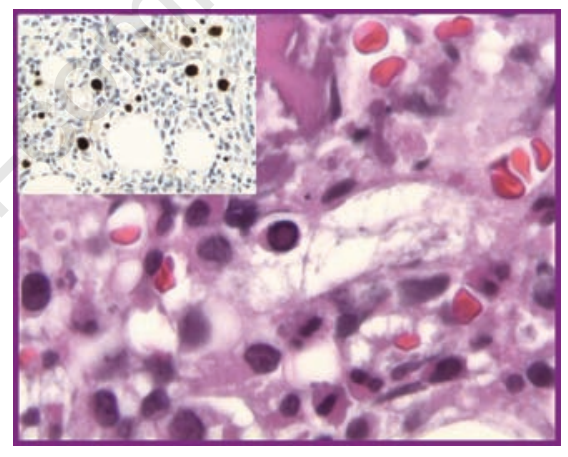

Figure 3. Parvoviral nuclear inclusions in erythroblasts seen in a bone marrow biopsy (hematoxylin and eosin stain; 400X magnification). Inset: parvovirus immunostain.

anemia, and a modest elevation in liver function and coagulation tests. Imaging studies showed a right inguinal abscess and massive hepatosplenomegaly (liver $24 \mathrm{~cm}$ and spleen $27 \mathrm{~cm}$ ). An ultrasound-guided liver biopsy revealed histological evidence of HSTL. Hematoxylin and eosin-stained sections of the liver revealed a diffuse sinusoidal infiltrate composed of intermediate cells with
Correspondence: Saadiya A. Haque, 3205 Halcyon Court, Ellicott City, MD 21043, USA

E-mail: saadiyahaque@yahoo.com

Key words: mature T-cell lymphoma, parvoviral infection, hepatosplenic lymphoma.

Conflict of interest: the authors report no conflicts of interest.

Received for publication: 7 February 2010. Accepted for publication: 5 May 2010.

This work is licensed under a Creative Commons Attribution 3.0 License (by-nc 3.0).

(C) Copyright S.A. Haque et al., 2010

Licensee PAGEPress, Italy

Pediatric Reports 2010; 2:e11

doi:10.4081/pr.2010.e11

medium-sized nuclei, loosely condensed nuclear chromatin, inconspicuous nucleoli, and pale eosinophilic cytoplasm (Figure 1). With immunohistochemistry staining, the atypical sinusoidal infiltrate was positive for LCA, CD3, CD7 (focal), CD43, CD56 (focal), and CD45R0. The neoplastic T-cell infiltrate was negative for the following markers: CD4, CD5, CD8, TdT, CD34, CD117, CD1a, myelo peroxidase, and B-cell antigens.

Bilateral bone marrow aspirates and biopsies revealed a mildly hypocellular marrow with trilineage hemotopoiesis and involvement by HSTL. Flow cytometric analysis also demonstrated the above characteristic profile, notably CD5 deletion, supporting the diagnosis. Based on flow cytometry on the bone marrow, the neoplastic lymphocytes expressed Tcell receptor gamma/delta and lacked the expression of T-cell receptor alpha/beta. Large circumscribed intranuclear viral inclusions were present in the erythroid precursors (Figures 2 and 3), confirmed as a parvovirus infection by immunohistochemical staining (Figure 3, inset). The inclusions were eosinophilic, with a ground-glass appearance, and were compressing the chromatin against the nuclear membrane. PCR analysis was positive for monoclonal T-cell receptor gamma chain gene rearrangement and negative for EBNA. With the patient's history of massive hepatosplenomegaly, these morphologic and immunophenotypic findings were indicative of a hepatosplenic T-cell lymphoma, gamma/ delta type, associated with parvoviral infection.

\section{Discussion}

This is the first reported case of HSTL with concomitant parvoviral infection. Most 
reported HSTL cases have been associated with EBV or HBV infections. HSTL is an aggressive disease with a poor prognosis and a median survival of less than two years. Effective therapeutic strategies have not been described, but proposed chemotherapeutic agents include platinum-cytabarine and pentostatin.

\section{References}

1. Vega F, Medeiros LJ, Gaulard P. Hepatosplenic and other gamma-delta T-cell lymphomas. Am J Clin Pathol 2007;127: 869-80.

2. Munir J, Preston G, Polish R. Case report: a common presentation of a rare disease hepatosplenic T-cell lymphoma. Hawaii
Med J 2004;63:341-3.

3. Beigel F, Jurgens M, Tillack C, et al. Hepatosplenic T-cell lymphoma in a patient with Crohn's disease. Nat Rev Gastroenterol Hepatol 2009;6:433-6.

4. Ozaki S, Ogasahara K, Kosaka M, et al. Hepatosplenic gamma-delta T-cell lymphoma associated with hepatitis B virus infection. J Med Invest 1998;44:215-7. 